\title{
La profillassi della nefrolitiasi tra evidenze, costi ed efficacia
}

\author{
G. Vezzoli
}

Unità di Nefrologia, IRCCS Ospedale "San Raffaele", Milano

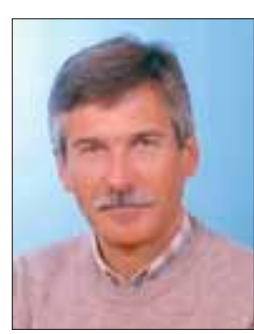

$\boldsymbol{L}$ prestazioni erogate dal SSN per la patologia nefrolitiasica sono in continuo aumento. Parte di queste spese è dovuta a recidive $e$ complicazioni. A vostro parere è possibile che una riallocazione di queste risorse verso la profilassi della malattia e delle sue recidive possa nel medio-lungo periodo rivelarsi cost-effective?

Diversi studi hanno dimostrato come la profilassi della litiasi renale sia economicamente vantaggiosa rispetto al semplice follow-up chirurgico (Pak CY. J Urol 1989; Parks JH and Coe F. Kidney Int 1996).

Se però il bilancio tra costi ed efficacia clinica viene affrontato da un punto di vista più ampio, cioè considerando quell'insieme di benefici, sociali, affettivi ed anche economici che la profilassi riesce a garantire, sono ancora più evidenti i suoi vantaggi, anche solo grazie al fatto di limitare la necessità di spiacevoli manovre chirurgiche seppur mini-invasive. La terapia profilattica ha in questo senso anche un ideale significato etico. Queste considerazioni dimostrano il valore della terapia profilattica. Dobbiamo però ammettere che pur dimostrando una riduzione delle recidive soprattutto nella calcolosi di calcio, gli studi sulle terapie profilattiche (diuretici tiazidici, citrato di potassio, allopiurinolo ...) sono limitati dalla bassa numerosità e dalla brevità del follow-up.

In sostanza esistono oggi sufficienti motivi per credere nell'efficacia della profilassi della nefrolitiasi. Tuttavia, nessuno a livello politico e amministrativo sembra avere intenzione di dedicarvi risorse.

Non ci resta che continuare a raccogliere elementi in favore della profilassi nella calcolosi renale. Probabilmente, per essere convincenti dovremmo costruire studi adeguati per disegno, numerosità e durata: ma chi è disposto oggi a garantire $\mathrm{i}$ fondi necessari per un simile studio?

Al medico è anche chiesto oggi di essere attento alle risorse economiche. Perciò, dovremmo sapere elaborare degli indicatori capaci di valutare l'efficacia clinica della profilassi della nefrolitiasi in relazione ai costi. Indici di efficienza ed efficacia sono disponibili per valutazioni a livello di macrosistema sanitario (Rutstein DD, et al, N Engl J Med 1976; 294: 582), ma non per singole malattie o territori. Vista la complessità dell' argomento, ci piacerebbe vedere uno sforzo per sviluppare la ricerca clinica in questo ambito da parte dalle amministrazioni pubbliche e degli organi politici.

Quanta parte della spesa per una gestione medica della nefrolitiasi grava sul paziente e quanto sul SSN nella vostra realtà regionale?

Mentre la terapia chirurgica della nefrolitiasi è in grandissima parte sostenuta dal SSN, la profilassi medica pesa prevalentemente sui risparmi del paziente. È però anche vero che la gestione medica della nefrolitiasi è ampiamente sottoutilizzata in Lombardia, come in tutte le altre Regioni. Sono infatti ancora pochi i pazienti che si rivolgono o vengono inviati al nefrologo per impostare la profilassi. 


\section{Giornale di Tecniche \\ Nefrologiche \\ \& Dialitiche \\ Anno XVI n ${ }^{\circ} 3$ \\ (C) Wichtig Editore 2004

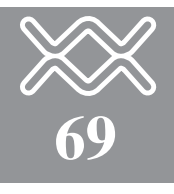

A fianco di provvedimenti quali l'idropinia e la dieta, il farmaco probabilmente più usato contro le recidive di nefrolitiasi è il citrato di potassio, che in Italia è però registrato tra gli integratori dietetici e non tra i farmaci di fascia A. I tiazidici e l'allopurinolo sono i soli farmaci di fascia A attivi nella prevenzione della litiasi. Paradossalmente però, se vogliamo essere rigorosi, i tiazidici e l'allopurinolo non hanno l'indicazione ministeriale per la nefrolitiasi, e quando usati per tale scopo dovrebbero perciò essere somministrati come farmaci di fascia C. Si può perciò concludere che non solo il costo della terapia è quasi sempre a carico del paziente, ma che il Sistema Sanitario Nazionale è quanto meno ingeneroso con i pazienti calcolotici. Sarebbe giusto e doveroso che questa situazione venisse riconsiderata.

È possibile prefigurare modelli organizzativi che consentano al paziente con NL di accedere a prestazioni del SSN, senza che questo comporti un eccessivo aggravio di spesa per la Regione?

I modelli organizzativi che sono stati fino ad oggi generalmente utilizzati, hanno previsto l'intervento non coordinato di due diversi specialisti. All'urologo spetta solitamente il primo approccio con il paziente con litiasi. In seguito, se l'urologo o il medico di famiglia sono attenti alla profilassi, oppure sono sollecitati in tal senso, inviano il paziente dal nefrologo.

Un'alternativa a questo approccio potrebbe consistere nella creazione di Centri regionali specializzati nella terapia della calcolosi. In questi Centri dovrebbero operare sia il nefrologo che l'urologo, in modo di unificare l'approccio alla malattia da parte dei due specialisti. Potrebbe essere così diminuito il numero delle visite e i tempi di attesa per i pazienti, nonché le spese per il SSN e per il paziente stesso.

Per costituire questi Centri, occorre però che sia urologo che nefrologo siano in grado di prescrivere la più opportuna terapia medica o chirurgica. Entrambi gli specialisti dovrebbe- ro perciò assumere informazioni e responsabilità che attualmente esulano dal loro interesse. Al di là della prescrizione terapeutica, i compiti dei due specialisti dovrebbero però restare distinti. Il nefrologo deve seguire il follow-up medico, mentre l'urologo deve dedicarsi alla gestione degli aspetti chirurgici.

Perché un simile modello organizzativo funzioni è però necessario che cresca la cultura medica relativa alla nefrolitiasi. Bisogna che i medici di famiglia inizino a considerare la nefrolitiasi come una malattia degna di follow-up clinico, e che la nefrolitiasi ritorni a ricevere l'attenzione dei nefrologi. Sono pochi infatti i Centri nefrologici che dimostrano oggi interesse verso la calcolosi renale. Lo sviluppo delle conoscenze in campo nutrizionale, metabolico e patogenetico ha aperto nuovi spazi culturali e clinici, mentre l'approccio familiare alla malattia può diventare importante per individuare i soggetti predisposti, ma non ancora affetti. Queste nuove prospettive si aggiungono ai classici aspetti metabolici e possono contribuire a rendere la nefrolitiasi meno arida di quanto apparso fino ad oggi a molti nefrologi e internisti.

A quali pazienti dovrebbe essere destinato lo studio metabolico della nefrolitiasi? $\grave{E}$ opportuno distinguere l'intervento in diversi livelli?

Uno screening per escludere le forme secondarie di calcolosi viene generalmente consigliato a tutti i pazienti fin dal primo episodio. Alcune linee guida hanno inoltre distinto livelli di intervento diagnostico in base al numero di recidive e consigliano uno screening metabolico completo solo nelle calcolosi ad alta recidivanza (più di due episodi in due anni). Anche se questo schema viene usato da molti medici e può apparire come appropriato, non mi ha mai convinto completamente. Compito del medico è infatti quello di evidenziare sin dal primo episodio i soggetti che tendono a recidivare sviluppando una vera malattia calcolotica. $\mathrm{Ci}$ sono tre ordini di fattori che dimostrano questa tenden- za. Il primo comprende i risultati dallo screening metabolico (Borghi L, et al. J Urol 1996; 155: 839, Strauss, et al. Am J Med 1981; 72: 17). Il secondo consiste nella familiarità per nefrolitiasi (Churan, et al. J Am Soc Nephrol 1997; 8: 1568). Il terzo comprende le abitudini alimentari (Churan, et al. New Engl J Med 1993; 328: 833). I dati a disposizione mostrano che una elevata escrezione di calcio e una bassa assunzione di fluidi individuano i soggetti più predisposti alle recidive. Anche altri difetti, come l'ipocitraturia, possono indurci a sospettare la tendenza alla recidiva, ma non ci sono studi specifici. La mia scelta personale è perciò quella di sottoporre i pazienti a screening metabolico fin dal primo episodio. Questa scelta è soprattutto importante in una dimensione ereditaria della malattia, perché alcune delle alterazioni che si trovano associate alla calcolosi (ipercalciuria, iperuricuria, ipocitraturia ...) possono essere trasmesse alla prole. E perciò importante conoscere queste alterazioni indipendentemente dalle recidive nei singoli soggetti. L'intensità della malattia calcolotica può infatti cambiare da soggetto a soggetto, anche all'interno della stessa famiglia, in relazione alle diverse abitudini dietetiche e alle diverse componenti genetiche acquisite dai genitori. È infatti la combinazione di più elementi che può influire sull'intensità della malattia calcolotica, producendo aspetti clinici differenti.

Quale secondo voi il ruolo del nefrologo e dell'urologo nel paziente affetto da NL? È opportuna una gestione della ESWL fatta direttamente dal nefrologo?

In linea generale bisogna ammettere che si è stabilito un certo scollamento tra l'intervento urologico e nefrologico, che ritengo sia il punto debole dell'intervento medico sul paziente nefrolitiasico.

Le due figure specialistiche dovrebbero interagire nella cura del paziente, senza che però siano perse la specificità dei loro ruoli: il nefrologo deve preoccuparsi prevalentemente della 
terapia farmacologica, mentre l'urologo delle procedure chirurgiche di diverso tipo. Questo non esclude che urologi preparati possano prescrivere la terapia medica. Così come non esclude la possibilità che il nefrologo possa eseguire trattamenti di litotrissia extracorporea, ma solo per i calcoli piccoli (inferiori al centimetro, con scarsa probabilità di complicanze) e nel quadro di una stretta collaborazione con gli urologi. Interventi più impegnativi su calcoli più complessi e le procedure endoscopiche non possono che essere di competenza del solo urologo, visto lo specifico bagaglio di conoscenze ed esperienze che comportano.

Oltre alla ESWL si sono diffuse negli ultimi anni procedure semi-invasive quali la ureteroscopia e la PUL. Ritenete opportuno che il nefrologo intervenga nella scelta delle procedure di trattamento dei calcoli?

Il nefrologo può certamente esprimere un parere sulla procedura chirurgica per il singolo paziente, anche se comunque la decisione sulla strategia di trattamento spetta in ultima istanza all'urologo, in quanto esecutore della manovra. Motivi di complessità clinica possono anche rendere indispensabile il giudizio dell'internista (il nefrologo rimane pur sempre un internista). Ovviamente questa interazione può meglio realizzarsi qualora il nefrologo abbia acquisito le conoscenze relative ai rischi e alle modalità dei trattamenti. Inoltre, questo scambio di pareri potrebbe più facilmente verificarsi se si realizzasse in un ambito organizzato di operativa collaborazione tra nefrologi e urologi.

Vi sono presso il vostro Centro esperienze di gestione integrata (nefrologo/urologo) della NL? Sareste in grado di proporre modelli organizzativi in tal senso?

A mia conoscenza ci sono oggi in Italia solo pochi Centri dove la collaborazione tra nefrologi ed urologi ha dato vita ad esperienze cliniche stabili.
Per quanto riguarda la nefrolitiasi, nell'ospedale dove opero la collaborazione tra $\mathrm{i}$ due specialisti è attiva anche se non rigorosamente coordinata. L'urologo dopo il trattamento chirurgico e prescrive una serie di esami ematici e urinari per la valutazione metabolica e li invia al nefrologo. Il nefrologo visita questi pazienti in un ambulatorio dedicato alla nefrolitiasi; con gli esiti degli esami già a disposizione può fornire indicazioni circa la terapia o la ulteriore diagnostica. Inoltre il nefrologo può anche prescrivere il trattamento extracorporeo, limitando però il proprio parere ai calcoli di dimensioni fino al centimetro e non complicati. Questa collaborazione rappresenta un passo avanti nella gestione dei pazienti, che seppur piccolo si è dimostrato estremamente utile per fare risparmiare tempo al paziente e per accelerare la definizione della terapia e della dieta più indicate. 